\title{
REDESCRIÇÃO DE NOMIMOSCOLEX ADMONTICELLIA (WOODLAND), COMB.N. (CESTODA: PROTEOCEPHALIDEA), PARASITO DE PINIRAMPUS PIRINAMPU (SPIX), UM SILURIFORME DE AGUA DOCE
}

\author{
Amilcar Arandas Rego ${ }^{1}$ \\ Gilberto Cezar Pavanelli ${ }^{2}$
}

\begin{abstract}
REDESCRIPTION OF NOMIMOSCOLEX ADMONTICELLIA (WOODLAND), COMB.N (CESTODA: PROTEOCEPHALIDEA) PARASITE OF PINIRAMPUS PIRINAMPU (SPIX), A FRESHWATER SILURIFORM FISH. The Monticelliidae species, Nomimoscolex admonticellia (Woodland, 1934), comb.n., parasite of Pinirampus pirinampu (Spix, 1829) a freshwater siluriform fish, is redescribed. This species is the type of the genus Myzophorus Woodland, 1934. The authors discuss the validity of this genus and propose its suppression. Their species are transferred to Nomimoscolex (Zigobothriinae) whose diagnose is modified to include: N. admonticellia, comb.n., N. pirarara (Woodland, 1935), comb.n. and N. woodlandi (Rego, 1984), comb.n.
\end{abstract}

KEY WORDS. Proteocephalidea, Nomimoscolex admonticellia, freshwater siluriform fishes

Os cestóides que parasitam peixes de água doce são ainda relativamente pouco conhecidos. No entanto, a taxonomia da Ordem Proteocephalidea foi em grande parte baseada na anatomia e morfologia destes helmintos. Woodland, a quem se deve o estabelecimento da taxonomia do grupo, descreveu, na década de 30, uma série de espécies, de material proveniente da Amazônia. Infelizmente, algumas dessas espécies, tendo sido descritas de modo inadequado, são hoje difíceis de serem identificadas. Tratam-se ainda, de helmintos que possuem escóleces extremamente pleomórficos, podendo se apresentar de várias formas, dependendo da fixação. Para melhor conhecimento das espécies de Cestoda, Proteocephalidea, parasitos de peixes sul americanos, torna-se necessário a sua revisão, utilizando novos espécimes corretamente fixados.

Obteve-se numerosos espécimes de cestóides do "barbado", Pinirampus pirinampu (Spix, 1829), procedentes dos Estados do Amazonas, Mato Grosso e Paraná, espécimes-tipo, depositados no The Natural History Museum, Inglaterra (BMNH), além de espécimes do Paraguai, depositados no Muséum d'Histoire Naturelle de Genève, Suíça.

1) Departamento de Helmintologia, Fundação Instituto Oswaldo Cruz, Caixa Postal 926, 20045-900 Rio de Janeiro, Rio de Janeiro, Brasil. Bolsista do CNPq.

2) Universidade Estadual de Maringá, Av. Colombo 3690, 87020-900 Maringá, Paraná, Brasil. Bolsista do CNPq. 
Estes parasitos foram identificados como Myzophorus admonticellia (Woodland, 1934). Note-se que o gênero Myzophorus Woodland, 1934 esteve sujeito a dúvidas desde que foi estabelecido. WOODLAND (1934) propôs $M$. admonticellia em Phyllobothridae (Tetraphyllidea), devido à distribuição peculiar da musculatura longitudinal dos proglotes (dois grupos de fibras longitudinais, sendo um externo e o outro interno) e também à forma unilaminar do ovário, além de outros caracteres menos importantes. Posteriormente, WOODLAND (1935) acrescentou três espécies ao gênero Myzophorus: $M$. pirarara de Phractocephalus hemiliopterus (Schneider, 1801), M. dorad de Brachyplatystoma rousseauxi ( $=$ B. flavicans Castelnau, 1855) e $M$. sudobim de Pseudoplatystoma fasciatus (Linnaeus, 1766). WOODLAND (1935) ampliou a diagnose de Myzophorus para incluir estas espécies, que exibiam caracteres nitidamente de Proteocephalidea.

WARDLE \& MCLEOD (1952) relacionaram Myzophorus entre os Tetraphyllidea.

YAMAGUTI (1959) transferiu Myzophorus para Endorchiinae, em Proteocephallidea.

Note-se que mesmo WOODLAND (1934) já tinha notado semelhanças entre Myzophorus e os Endorchiinae.

FREZE (1965), em sua monumental revisão dos Proteocephalidea, discutiu criticamente os caracteres de Myzophorus, considerando-os de pouco valor para a sistemática. FREZE, no mesmo trabalho, transferiu Myzophonıs sudobim Woodland, 1935 para o gênero Nomimoscolex, em Zigobothriinae. Como o nome sudobim estava ocupado neste gênero, criou um novo nome para a espécie, Nomimoscolex woodlandi Freze, 1965.

Posteriormente, REGO (1987) criou o gênero Houssayela para esta espécie, que passou a chamar-se Houssayela sudobim (Woodland, 1935), revalidando o nome original sudobim. REGO (1984) descreveu Myzophorus woodlandi, parasita de Phractocephalus hemiliopterus (Schneider, 1801) da Amazônia. Em trabalho recente REGO (1991) colocou Myzophorus dorad Woodland, 1935 na sinonímia de Nomimoscolex piraeeba Woodland, 1934, ambas parasitas de Brachyplatystoma filamentosum (Lichtenstein, 1819). No mesmo trabalho REGO transferiu Myzosphorus de Endorchiinae para Zigobothrïnae, devido a supressão da subfamília Endorchïnae, já que as espécies pertencentes a Endorchiinae possuem características semelhantes as de Zygobothriinae.

PAVANELLI \& MACHADO DOS SANTOS (1991) referiram, sem descrição, Myzophorus schaefferi em Pinirampus pirinampu (Spix, 1829) do rio Paraná. Tendo oportunidade de reexaminar estes espécimes, concluiu-se tratarse de M. admonticellia. Na ausência de descrição, Myzophorus schaefferi deve ser considerado nomem nudum.

A situação do gênero Myzophorus é controversa. Com a finalidade de esclarecer o valor de seus caracteres, apresenta-se a sua redescrição, utilizando novos espécimes de $M$. admonticellia e os espécimes-tipo do The Natural 
History Museum, Inglaterra.

\section{MATERIAL E MÉTODOS}

Os helmintos foram fixados pelo formol a $5 \%$ a frio ou aquecido e conservados em álcool 70\%. Para montagem em lâminas, os helmintos foram corados pela hematoxilina Delafield ou carmim clorídrico alcoólico de Langeron; desidratados e passados pelo creosoto de faia e montados em bálsamo do Canadá. Secções do estróbilo com $8 \mu \mathrm{m}$ foram coradas pela hematoxilinaeosina. Os desenhos foram feitos com auxílio de câmara clara e as medições são apresentadas em micrometros, com as médias entre parênteses.

\section{RESULTADOS}

Com a supressão do gênero Myzophorus tornou-se necessário ampliar a diagnose de Nomimoscolex para receber estas espécies.

Nomimoscolex Woodland, 1934: Zygobothriinae de tamanho pequeno ou médio. Estróbilo acraspédote. Escólex sem metascólex, com ventosas comuns e salientes. Região apical, glandular, pode estar presente. Proglotes, em geral mais longos que largos. Sistema reprodutivo medular, mas o ovário e o útero podem ter pequenas porções no córtex. Útero com numerosos divertículos. Musculatura longitudinal pode ser conspícua ou inconspícua. Glândulas vitelogênicas laterais ou em crescente, em cortes. Parasitas de peixes de água doce da Ámerica do Sul.

\section{Nomimoscolex admonticellia (Woodland, 1934), comb.n.}

Figs 1-7

Proveniências: rio Amazonas (localidade-tipo); Itacoatiara, rio Amazonas, Amazonas; rio Cuiabá, Mato Grosso; rio Paraná, Paraná; rio Paraguay, Paraguai.

Material depositado na Coleção Helmintológica da Fundação Instituto Oswaldo Cruz (FIOCRUZ) sob os números: 33.031a, c; 33.032a, e e 33.033a, b e BM(NH) 1965.23.119-138.

Descrição (baseada em dez exemplares). Helmintos de tamanho médio, o maior estróbilo mediu 117 e o menor 52 . Cerca de 120 proglotes; a maioria maduros ou grávidos. Segmentação nítida. Acraspédotes. Proglotes grávidos, de 221x122 a 371x97 (278x101).

Escólex pleomórfico, sem metascólex, ventosas comuns e salientes. $\mathrm{Na}$ região apical de natureza glandular, não visível em alguns escóleces. O escólex mediu 35x47 a 67x67 (50x58). Ventosas esféricas, diâmetro 21 a 30 (25). Relação escólex: ventosas (média das medidas do escólex e ventosas) =1:2,25.

Os poros genitais alternam de modo irregular; abrem no 1/2.25 anterior nos proglotes. A bolsa do cirro ocupa cerca de $1 / 3$ da largura dos proglotes, mediu 23x11 a 37x14 (31x14). Canal deferente enovelado. A vagina abre no átrio, 

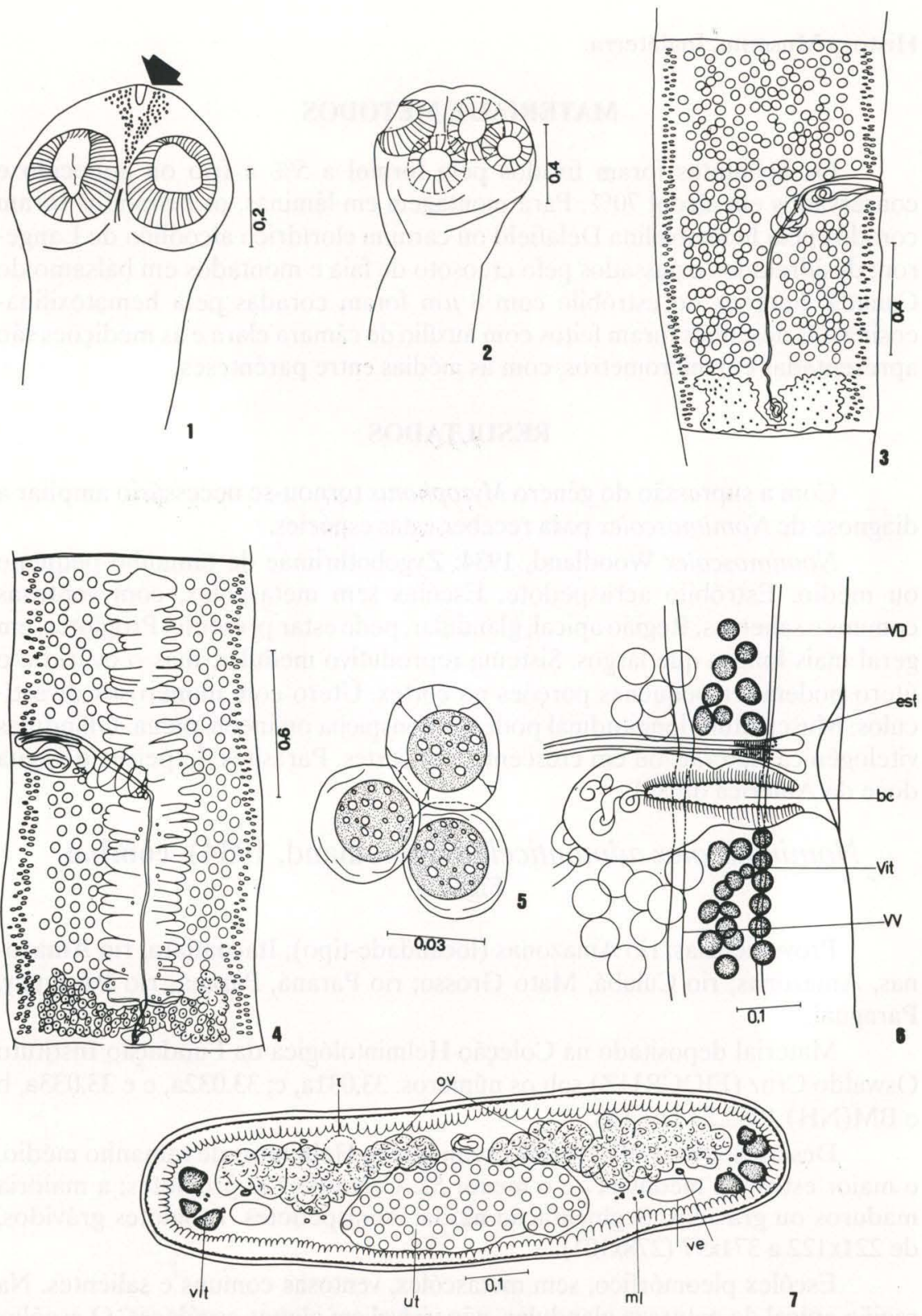

Figs 1-7. Nomimoscolex admonticellia (Woodland, 1934), comb.n. (1-2) Escólex, note a glândula apical (seta); (3) proglote maturo e (4) grávido; (5) ovos, dispostos em grumos; (6) detalhe da região do átrio genital: (vd) vaso excretor dorsal, esfíncter vaginal (esf), bolsa do cirro (bc), glândulas vitelogênicas (vt), testículos (t) e vaso excretor ventral (vv); (7) corte tranversal de proglote grávido: ovário (ov), útero (ut), músculos longitudinais ( $\mathrm{ml}$ ), glândulas vitelogênicas (vit), vasos excretores dorsal e ventral (vv). 
anteriormente à bolsa; possui esfíncter vaginal pouco nítido, além de células cromófilas junto ao átrio. Cerca de 300-400 testículos, que mediram 50-80 (64) de diâmetro, estando dispostos de modo contínuo nos proglotes. Ovário bilobado, a largura variou, 58 a 96 (65). O útero, nos últimos segmentos, apresenta 50-60 divertículos curtos de cada lado do eixo uterino. Não foram observados ganchos nos ovos; a membrana externa é frágil, mediu 31 de diâmetro e a interna, 21. As glândulas vitelogênicas são constituídas por numerosos folículos laterais, em toda a extensão dos proglotes. Em cortes histológicos foram observadas células subcuticulares muito desenvolvidas. A musculatura longitudinal é inconspícua; poucas células musculares, possivelmente mioblastos, são observadas, dispersas irregularmente. Apenas em cortes sagitais foi possível observar delicadas fibras isoladas, delimitando os vitelinos corticais da medula. Pequenas porções do ovário penetram na região cortical.

\section{DISCUSSÃO}

Há algumas diferenças entre os espécimes ora descritos e o que foi descrito por WOODLAND (1934), como exemplo, o maior número de testículos (WOODLAND referiu apenas 200). Estas diferenças podem ser atribuídas ao mal estado de fixação que os espécimes apresentavam, não permitindo a observação de determinadas estruturas. WOODLAND também não referiu a presença de sulcos transitórios no escólex, prováveis artefatos pós-fixação, bem como não descreveu o ápice glan'́ular que observamos. Estas estruturas apicais não são tão raras em Monticelliidae. PAVANELLI (1991) e REGO (1991) constataram estruturas similares em Monticellia coryphicephala (Monticelli, 1891) e Nomimoscolex piraeeba (Woodland, 1934), respectivamente.

WOODLAND (1934) descreveu Myzophorus admonticellia em Phyllobothriidae (Tetraphyllidea), impressionado pelas características da musculatura longitudinal, que considerou semelhantes a dos Tetraphyllidea; além de outros caracteres menos importantes, como a forma do ovário. Em trabalhos anteriores REGO $(1984 ; 1991 ; 1992)$ criticou a idéia da existência de Tetraphyllidea em peixes Siluriformes de água doce. Alguns caracteres, aparentemente de Tetraphyllidea, não se afastam do que é comumente encontrado em Proteocephalidea; no máximo tratar-se-ia de evolução paralela ou convergente.

De acordo com WOODLAND (1934), M. admonticellia possuiria músculos longitudinais constituídos por duas camadas, externa e interna, ainda que pouco conspícuos. Nos cortes que examinou-se, não foi possível observar músculos longitudinais organizados, apenas raríssimas fibras isoladas, separando as glândulas vitelogênicas dos campos testiculares, que serviu para confirmar a espécie em Zygobothriinae. Note-se que o próprio WOODLAND somente pode observar músculos em estróbilos contraídos; em espécimes distendidos a sua observação é quase impossível. Por outro lado, o exame dos tipos no BMNH não confirmou a descrição de WOODLAND.

FREZE (1965) criticou a maioria dos caracteres de Myzophorus. Ele considerou como tendo pouco valor taxonômico a questẩo da textura dos 
parênquimas corticais e medulares, o pseudo-metascólex e a disposição em crescente das glândulas vitelogênicas. FREZE considerou apenas bom caráter a disposição especial da musculatura, descrito por WOODLAND.

Como Myzophorus foi descrito com base na presença da musculatura longitudinal em duas camadas, caráter de difícil demonstração, é melhor suprimir o gênero. O próprio WOODLAND (1934) fez notar o absurdo de que apenas algumas fibras musculares possam determinar a posição taxonômica de um helminto.

Propõem-se pois, que as espécies remanescentes de Myzophorus Woodland, 1934 sejam transferidas para o gênero Nomimoscolex Woodland, 1934 (Zygobothriinae), com as seguintes combinações: N. admonticellia (Woodland, 1934), comb.n., N. pirarara (Woodland, 1935), comb.n., $N$. woodlandi (Rego, 1984), comb.n. O gênero Nomimoscolex tem ampliada sua diagnose para abranger estas espécies.

AGRADECIMENTOS. Ao Dr. David I. Gibson, pela cessão de espécimes tipos de Woodland, depositados no The Natural History Museum, Inglaterra, e ao Dr. Alain de Chambrier, do Muséum d'Histoire Naturelle de Genève, Suíça, pela cessão de espécimes de proteocefalídeos do Paraguai.

\section{REFERÊNCIAS BIBLIOGRÁFICAS}

FREZE, V.I. 1969. Principles of cestodology, vol. 6. In: K.I. SKRJABIN (ed.) Proteocephalata cestodes of fishes, amphibia and reptiles. Moscou, Programa de Traduções Científicas de Israel, 538p.

PAVANELLI, G.C. 1991: Proteocephalidae (Platyhelminthes - Cestoda) parasitos de peixes, em especial, Pimelodidae, do rio Paraná, Brasil. Tese de Doutorado, não publicada, Universidade Federal do Paraná, Curitiba, 148p.

PAVANELLI, G.C. \& M.H. MACHADO DOS SANTOS. 1991. Proteocefalídeos parasitos de peixes, em especial pimelodídeos do rio Paraná, Paraná. Rev. Unimar, Maringá, 13 (2): 163-175.

REGO, A.A. 1984. Proteocephalidea from Amazonian freshwater fishes: new systematic arrangement for the species described by Woodland as Anthobothrium (Tetraphillidea). Acta Amazonica 14 (1/2): 86-94.

1987. Cestóides proteocefalídeos do Brasil. Reorganização taxonômica. Rev. Bras. Biol. 47 (1/2): 203-212.

1991. Redescription of Nomimoscolex piraeeba Woodland, 1934 (Cestoda, Proteocephalidea) from the Amazon catfishes, Brachiplatystoma spp. with proposal of synonyms and invalidation of Endorchiinae and Endorchis. Mem. Inst. Oswaldo Cruz 86 (2): 229-232.

1992. Redescription of Gibsoniela mandube (Woodland, 1935) (Cestoda, Proteocephalidea), a parasite of Ageneiosus brevifilis (Pisces, Siluriformes), and reapraisal of the classification of the Proteocephalideans. Mem. Inst. Oswaldo Cruz 87 (3): 417-422. 
WARDLE, R.A. \& J.A. MCLEOD. 1952. The Zoology of Tapeworms. Minneapolis, The University of Minessota Press, 780p.

WOODLAND, W.N.F. 1934. On the Amphilaphorchidinae, a new subfamily of Proteocephalid cestodes and Myzophorus admonticellia, gen.n., parasite in Pinirampus pirinampu from the Amazon. Parasitology 26: 141-149.

1935. Additional cestodes from the Amazon Siluroids Pirarará, Dórad and Sudobim. Proc. Zool. Soc. London, part IV: 851-864.

YAMAGUTI, S. 1959. Systema Helminthum. 2. The cestodes of vertebrates. New York, Intersc. Publ. Inc., 860p.

Recebido em 28.II.1993; aceito em 15.VIII.1993. 